\title{
Modelos contemporáneos de intervención en Trabajo Social: revisión bibliográfica del modelo narrativo*
}

\author{
Alexis Bustos Villarroel $^{* *}$
}

\section{RESUMEN}

El presente trabajo es fruto de la recopilación bibliográfica respecto de los modelos de intervención en Trabajo Social, cómo ellos se han reformulado, conforme han ido confluyendo los distintos cambios de paradigmas, los cambios en las cosmovisiones filosóficas y finalmente, en las teorías que sostienen dichos modelos, o sea, en las distintas epistemologías ${ }^{1}$. El presente artículo inicia con un análisis respecto de las influencias que ha tenido la ciencia formal, en el desarrollo de los modelos y teorías de las ciencias sociales y ciencias aplicadas en general. De esta forma, se analizan brevemente los distintos modelos de intervención en trabajo social, finalizando en los planteamientos del Asistente Social australiano Michael White y el Antropólogo neozelandés David Epston, precursores de la Terapia Narrativa.

Palabras clave: Cambios de Paradigma, Ciencias Formales, Ciencias Sociales, Trabajo Social y Terapia Narrativa.

* Artículo recibido: 02/06/2014. Artículo aprobado: 22/07/2014. Versión final: 12/10/2014.

" Chileno. Trabajador Social. Postítulo en Psicología, Familia y Derecho; Postítulo en Psicología Jurídica al Servicio de la Investigación Forense y Criminal. Terapeuta Familiar Sistémico, Formación (c) en Terapia Narrativa. Adscrito a la Red Comunal Por La No Violencia, Valparaíso, Chile. Actualmente, Trabajador Social en Programa de Reparación De maltrato Grave y abuso Sexual, PRM CAVAS Regional de Valparaíso, Chile, convenio SENAME-PDI. Correo electrónico: bustosvillarroel@gmail.com

1 El concepto de Epistemología ha de ser comprendido, desde los planteamientos del Antropólogo Gregory Bateson, no en el sentido tradicional filosófico que versa sobre el estudio del método científico y su producto. A este respecto y siguiendo los planteamientos de Bateson (1976), entenderemos la epistemología de tres formas particulares; como una forma de explicar y comprender el mundo; con las reglas que se han de utilizar para otorgar sentido al mundo, para que exista una coherencia con los seres humanos; finalmente, como las operaciones que utilizan las personas para conocer, pensar y decidir. 


\title{
Modelos contemporâneos de intervenção em Trabalho Social: Revisão Bibliográfica do Modelo Narrativo
}

\begin{abstract}
RESUMO
O presente trabalho é fruto da compilação bibliográfica referente aos modelos de intervenção em Trabalho Social, como eles, tem sido reformulados conforme têm ido concorrendo as diferentes mudanças de paradigmas, mudanças nas visões filosóficas e finalmente, nas teorias que sustentam esses modelos, ou seja, nas diferentes epistemologias. O presente artigo começa com uma análise respeito das influências que tem a ciência formal no desenvolvimento dos modelos e teorias das ciências sociais e ciências aplicadas em geral. Desta forma, analisam-se brevemente os diferentes modelos de intervenção em trabalho social, finalizando nas abordagens do trabalhador social australiano Michael White e o antropólogo Neozelandês David Epston, pioneiros da terapia narrativa.
\end{abstract}

Palavras-chave: Mudanças de Paradigma, Ciências Formais, Ciências Sociais, Trabalho Social e Terapia Narrativa.

\section{Contemporary Models of Social Work Intervention: Review of the Narrative Model}

\begin{abstract}
This work is the output of the bibliography compilation regarding intervention models in social work and how they have been reformulated according to new and different changes in paradigms, in philosophical worldviews and finally, in theories supporting those models, that is, at different epistemologies. This article begins with a discussion regarding the influences that formal science have had in the development of models and theories in social sciences and applied sciences in general. It briefly discusses the different models of social work intervention and finishes with the approach of the Australian social worker Michael White and the Zealander anthropologist David Epston as precursors of the Narrative Therapy.
\end{abstract}

Keywords: Paradigm changes, formal sciences, social sciences, social work and narrative therapy.

\section{El Trabajo Social y su relación con el desarrollo de las Ciencias sociales}

Respecto del fenómeno estudiado y en relación con la generación de conocimiento, podemos informar que la historia de la humanidad y el desarrollo de las formas de conocer y explicar los fenómenos, han estado caracterizadas por tres epistemologías 
que han permeado en menor o mayor grado las ciencias sociales en general. De esta manera, lo lineal-causalista (positivista), lo sistémico-circular (teoría general de sistemas) y la cibernética de segundo orden (constructivista), obedecen a cambios de paradigmas al interior de un cuerpo de conocimientos sistematizados, los cuales podríamos llamar ciencia, profesión o teorías (Demicheli, 1995). Estas epistemologías han influido en distintos grados, en la estructuración de la ciencia formal o las denominadas ciencias duras, pero también, poseen una influencia importante en el desarrollo de las ciencias sociales.

En el acápite anterior, se esboza cómo se han desarrollado las ciencias sociales en base al desarrollo de las llamadas ciencias exactas o ciencias duras. Pero no sólo las ciencias se han desarrollado en esa dirección, las ciencias sociales en general: la Sociología, Economía, Antropología, Derecho y sobre todo la Psicología, han transitado el largo camino, comenzando por el positivismo, llegando a la vinculación con la teoría general de sistemas y finalmente en lo que conocemos como constructivismo ${ }^{2}$. Prosiguiendo con el planteamiento anterior, las ciencias aplicadas o la tecnología social, no ha quedado exenta del desarrollo del pensamiento científico y epistemológico.

Suele existir una confusión entre lo que es el Constructivismo y el Construccionismo. En lo que respecta al constructivismo, exististe consenso que emerge como epistemología ligada al desarrollo de la cibernética de segundo orden. Y a las contribuciones de los biólogos chilenos Humberto Maturana y Francisco Varela, conjuntamente a los planteamientos desarrollados por los físicos Heinz Von Foester y John Von Neumann, entre otros autores prominentes en el desarrollo de dicho conocimiento. La cibernética de segundo orden o, como se llamaría más tarde, constructivismo, emerge en escena en momentos que, los autores comenzaron a preguntarse por el lugar del observador, hasta ese entonces se consideraba que el lugar del observador, estaba "fuera" de los sistemas que se observaban y por ende, no tenían mayor influencia en los objetos que se observaban en un sistema cualquiera. Cuando la cibernética desborda su campo de estudio y comienza a cuestionarse respecto del observador, es que emerge el constructivismo como epistemología, en la cual el observador se encuentra inserto en el sistema observado y por lo tanto, influye en las observaciones, prescripciones y descripciones. De esta forma, comienza la crisis al conocimiento objetivo. Por su parte, premisas fundamentales del construccionismo social, se basan en los planteamientos de Kenneth Gergen (1985, 1991, 1994, 1999), los cuales versan sobre los siguientes tópicos; 1) Salir de la razón individual y llegar a la retórica comunal; 2) Ir de un mundo objetivo a uno construido socialmente, y 3) Superar una concepción de lenguaje representativo y ahondar un modo de lenguaje entendido como una práctica pragmática. De igual forma, retomar planteamientos posmodernistas respecto de la "caída" de la objetividad y la noción de verdad entre las ciencias sociales, sería una mera falacia argumentativa carente de sentido. 
De esta forma, autores como Aylwin y Solar, sostienen que el trabajo social se ha nutrido de este desarrollo científico y teórico, es más, el trabajo social se ha desarrollado en paralelo con los cambios epistemológicos en la física, la biología, la filosofía, la lingüística, la antropología, la sociología, la psicología y en casi todo el campo del conocimiento social y científico (Aylwin y Solar, 2002: 108).

Sumado a ello, el trabajo social también se he permeado de pensamientos filosóficos y de ciertos paradigmas específicos. Así, siguiendo los planteamientos de José Morán Carrillo³ , la disciplina ha transitado -al alero de la filosofía y los paradigmas- por el paradigma funcionalista, que retoma planteamientos sociológicos positivistas y modernistas. También lo ha hecho por el paradigma conflictivista, basado en los planteamientos de Marx y Engels, la sociología crítica, la reconceptualización del trabajo social y la emergencia de formas radicales del trabajo social, ligadas a ideologías feministas, liberales, socialistas y ecologistas. Finalmente concluye en la relación del trabajo social con el paradigma hermenéutico o interpretativo, basado en los planteamientos del interaccionismo simbólico de Blummer, la fenomenología de Husserl, la sociología del conocimiento de Schütz, la etnometodología de Garfinkel, la construcción social de la realidad de Berger y Luckmann, entre otros autores fecundos en teoría social postmoderna, en la cual no interesa el conocimiento objetivo, la búsqueda de la verdad o la explicación del fenómeno (Morán Carrillo, 2003). Claramente podemos apreciar en la revisión sistemática que efectúa Morán, el tránsito entre el positivismo, la teoría general de sistemas y el constructivismo.

Finalmente, la autora Teresa Matus (1999), en su libro Propuestas Contemporáneas en Trabajo Social, efectúa un análisis minucioso del impacto que ha tenido el positivismo en relación con el trabajo social, cómo ello generó y genera ciertas nociones de la importancia de las explicaciones teóricas, las cuales a veces se distanciaban enormemente de la realidad observada. La necesidad de uno o varios métodos de intervención científicamente validados, la concepción de verdad y objetividad, la posición de "experto" en la cual el/la trabajador(a) social, tenía las respuestas

Sociólogo y Trabajador Social español. 
adecuadas para la vida del otro $u$ otra, entre otras nociones ligadas al positivismo.

Luego de ello, comienza a gestarse la crisis intelectual y teórica que culmina en la reconceptualización y cómo los antiguos "modelos" heredados del positivismo, del análisis fragmentario de la realidad social, de la cuantificación y la búsqueda de explicaciones universales, comenzaban a perder sentido. De esta manera, la tensión entre la teoría y la práctica hace su aparición en escena, tensión que aún no está resuelta.

Finalmente, el trabajo social se relaciona con la postmodernidad, sumándose a los planteamientos de Derrida, Lyotard, Foucault, entre otros, donde la premisa fundante es la desconfianza de "relatos metanarrativos de valor universal y cuestiona la capacidad de alcanzar una verdad que no sea relativa en un contexto parcial y localizado" (Matus, 1999: 128). Entonces, la semejanza entre el postmodernismo y el constructivismo radica en que conceptos como "verdad" y "objetividad" no tendrían sentido; sumado a ello, la importancia del conocimiento localcomunitario subjetivo, en contraposición al conocimiento universal, global-explicativo.

Se ha podido apreciar, de manera sucinta, cómo el trabajo social se ha imbricado con el desarrollo de las ciencias y las CCSS, cómo los distintos cambios de paradigmas filosóficos e ideológicos han sido capaces de permear la praxis profesional, así como también, qué ha significado estar en los tres momentos más importantes del desarrollo del conocimiento: Positivismo, Teoría General de Sistemas y Constructivismo. Para mayor desarrollo de estas ideas, continuaremos profundizando sobre el desarrollo de los modelos de intervención, en la base del tránsito antes mencionado.

\section{Desarrollo de los modelos de intervención en trabajo social}

Existe consenso en admitir que, los modelos de intervención en trabajo social reconocidos y con mayor frecuencia de uso, son los que se aprecian a continuación. Autores como Aylwin y Solar (2002), Camparini y Luppi (1991), Quintero (2004) y De Robertis (1994 y 2006), entre otros, reconocen los siguientes: 


\section{Modelos Clásicos de Intervención en Caso-Familia:}

- Psicosocial: (1937 y ss.) Inmerso en el Casework y en los inicios de la profesionalización de la disciplina.

- Resolución de Problemas: (1940) Resolver problemas puntuales con el objeto de superar las frustraciones iniciales.

- Intervención en Crisis: Intervención de una crisis puntual y resolución de ella.

- Terapia Familiar: (1950 y ss.) Modelo ecléctico, se basa en principios sistémicos, transgeneracionales y estructurales de la familia.

- Centrado en Tareas: (1965 y ss.) Inmerso en el trabajo social comunitario, se basa en que la persona define e identifica la problemática a trabajar.

- Ecológico: (1980 y ss.) Se basa en la premisa del hombre en constante desarrollo, en directa relación con el ambiente social circundante.

Modelos Contemporáneos de Intervención en CasoFamilia:

(Modelos de primera onda ${ }^{4}$ )

- Sistémico: Basado en la premisa de la importancia de las relaciones sociales, familiares y personales, las cuales influyen o son de influencia en un sistema dado.

- Fenomenológico: Premisas básicas se fundan en la fenomenología del observador, no generar problemas sociales donde no los hay, observar y analizar el fenómeno social, conducente a una posible resolución del mismo (importancia del otro como un legítimo otro).

\section{(Modelos de segunda onda)}

- Enfoque Social-Sistémico: (Ralph E. Anderson e Irl Carter) Basado en la teoría de sistemas, en conjunto con bases sociológicas (Weber, Mead y Blumer) y los avances propios del de-

\footnotetext{
La denominación de modelos de primera y segunda onda, es una denominación del autor, ella no se encuentra sostenida en los textos de trabajo social consultados. Dicha nomenclatura obedece al intento de situar los distintos modelos en la modernidad y la posmodernidad. Así como los modelos clásicos y contemporáneos, se encuentran en estricta relación con el desarrollo del positivismo, teoría general de sistemas y constructivismo.
} 
sarrollo mismo de la teoría de sistemas aplicada a la sociedad y las problemáticas sociales.

- Servicio Social y Modelo Sistémico: (Anna María Campani$\mathrm{ni}^{5}$ y Francesco Luppi $^{6}$ ) Síntesis teórica conceptual de la teoría de sistemas aplicada en el servicio social, influencias del modelo de Millán.

- Trabajo Social Clínico: (Amaya Ituarte Tellaeche ${ }^{7}$ ) Forma especializada del Trabajo Social, que siguiendo el procedimiento científico y por medio de un proceso psicoterapéutico trata de ayudar a las personas, familias o grupos pequeños que se encuentran en situaciones de conflictos (influencias del positivismo y el modelo médico).

- Terapia Familiar: (Joseph Pérez ${ }^{8}$ ) La Terapia Familiar como una modalidad para la intervención con familias. Define a la Terapia Familiar como "el proceso interactivo que busca ayudar a la familia a recobrar la homeostasis, con la cual todos su miembros se sientan a gusto" (Pérez, J., 1984: 55).

- Proceso de Cambio: (Peggy Papp ${ }^{9}$ ) Los conceptos clave del pensamiento de sistemas están relacionados con la integridad, la organización y la regulación. Importancia de la relación existente entre la cognición, ideación y lo conductual.

- La Voz Terapéutica: (Olga Silverstein ${ }^{10}$ ) Centrado en las observaciones y descripciones de las personas, para lo cual distingue una serie de dominios interaccionales, descriptivos y observacionales. Por su parte, los marcos de observación, políticos y semánticos enmarcadas en la terapia sistémica, con acento en las observaciones (descripciones) que se realizan. (Keeney y Ross) son la base cibernética de primer y segundo orden.

- Proceso de validación Humana: (Virginia Satir ${ }^{11}$ ) Se basa en premisas sistémicas y humanistas: en la base de la intervención está la concepción de que cada persona tiene el potencial de cambiar y de aprender, todas las personas tienen recursos y el terapeuta está al servicio de la persona (posición de no-experto).

Asistente Social y Terapeuta Familiar italiana.

Psicólogo y Terapeuta Familiar italiano.

Trabajadora Social española.

Trabajador Social, Psicólogo Clínico y Terapeuta Familiar estadounidense.

Trabajadora Social y Terapeuta Familiar estadounidense.

Trabajadora Social y Terapeuta Familiar estadounidense.

Trabajadora Social y Terapeuta Familiar estadounidense. 
- Intervención Familiar en Trabajo Social: (Vesna Tomic ${ }^{12}$ ) Bases fundantes en la teoría de sistemas y teoría ecológica. Importancia de la relación entre los sujetos-sociales y las instituciones-sociales. Ambos portadores de recursos y necesidades, el trabajador social es un intermediario de las partes.

- La Red Invisible: (Marianne Walters ${ }^{13}$ ) Enfoque de familia patriarcal, complementariedad de roles en la base de la intervención. Modelo feminista, se busca la simetría de los roles en la familia.

\section{Modelos Emergentes en Trabajo Social de Caso-Familia:}

- Narrativo: (Michael White ${ }^{14}$ y David Epston ${ }^{15}$ ) Principios fundamentales en la imposibilidad de acceder a la realidad (Bateson) influencias del poder en las personas (Foucault), analogía del texto (Derrida). Generan un espacio de intervención en el cual las historias son las que moldean las vidas de las personas, existiendo historias subyugadas o secundarias, las cuales posibilitan el engrosamiento de la trama dominante. Externalización de los problemas como premisa fundamental

- Cibernética de primer orden:

- Cibernética de segundo orden (Constructivista):

- Construccionista Social / Socioconstruccionista:
Los autores lo han llamado el paradigma de la complejidad, para referirse a la Cibernética, Constructivismo y Construccionismo Se han efectuado trabajos a nivel metateórico, según lo investigado; no existen aún propuestas o modelos de intervención que oscilen en estas teorías.

Es importante mencionar que cada uno de los modelos expuestos anteriormente, fue configurándose como parte de un contexto histórico, político, social y cultural que posibilitó la emergencia de dicho modelo; cada uno de ellos desempeñó y desempeña un rol importante en el contexto de la praxis social inherente al trabajo social. Es importante también mencionar que, por las limitaciones de espacio, no se puede ahondar en la emergencia de cada uno de los modelos mencionados.

\footnotetext{
Trabajadora Social y Terapeuta Familiar chilena.

Trabajadora Social y Terapeuta Familiar estadounidense.

Asistente Social y Terapeuta Familiar australiano.

Licenciado en Antropología y Sociología, Certificado de Capacitación en Trabajo Social neozelandés.
} 


\section{Terapia narrativa}

La Terapia Narrativa es propuesta por el Asistente Social australiano Michael White y el Antropólogo y Terapeuta Familiar neozelandés ${ }^{16}$ David Epston, quienes en 1980 formalizaron sus ideas en un libro llamado "Medios Narrativos para fines terapéuticos". Ambos autores, por separado y luego en conjunto, se encontraban trabajando con ideas "poco ortodoxas"17 en el campo de la terapia familiar. De esta forma, uno de los planteamientos centrales en la práctica narrativa, es la externalización de los problemas. El problema ya no es la persona o la relación, sino, el problema es el problema. De esta forma, al separar el problema de la persona, se genera un espacio simbólico de control y manejo de la situación problemática.

Los aspectos fundamentales de la Terapia Narrativa, son los siguientes:

\section{Analogía del Texto}

Desde la práctica narrativa, la persona es considerada como una posibilidad de cambio constante, en tanto no estaría definida ni por sus condicionantes biológicos, sociales o culturales, sino la persona estaría íntimamente relacionada con su historia y las historias que cuentan de él o ella. De igual forma, cómo

Si bien David Epston posee nacionalidad neozelandesa, es originario de Canadá.

17 Por ideas "poco ortodoxas" se hace referencia, en primera instancia, a las disciplinas que representan David Epston y Michael White, quienes no pertenecen al campo de la psicología o psiquiatría, desarrollando históricamente modelos, técnicas y teorías en el campo de la terapia familiar. Si bien tanto en Australia como en Nueva Zelanda existe apertura a quienes quieran perfeccionarse como terapeutas familiares, las propuestas en dicha área provenían -hasta ese entonces-, de profesionales de la psicología y la psiquiatría. En segunda instancia, las ideas propuestas por David Epston se relacionaban con los ritos de pasaje propuestos por Van Gennep, trabajados por el antropólogo Víctor Turner, y la comprensión de los fenómenos de Clifford Geertz (Antropólogo). Por su parte, Michael White estuvo interesado en la epistemología de Gregory Bateson (Antropólogo), los planteamientos generales del filósofo Michel Foucault, los planteamientos de Jerome Brunner (Psicólogo y Pedagogo) respecto de su teoría en general; Jacques Derrida, filósofo, de quien retoma los planteamientos acerca de la deconstrucción y la crítica textual. Como se aprecia, los autores basales en los que se apoyan Epston y White, se relacionan con campos de acción distintos de la psicología y la psiquiatría, disciplinas dominantes hasta el momento en terapia familiar. Son "poco ortodoxas" por el hecho de incluir aspectos socioculturales, contextuales y políticos al espacio terapéutico y sobre todo, por distanciarse del positivismo (el problema es la persona), de la teoría de sistemas (el problema es la relación) y quedarse en el postmodernismo-constructivismo (el problema es el problema). 
esas historias moldean su vida. Este sujeto histórico-contextual ha de ser considerado como un texto, como posibilidad abierta de ser leída, releída, escrita y reescrita. Ello posibilita la revisión histórico-vital del ser, en la cual se encuentran otras versiones de sí mismo, las historias no dichas o "irrelevantes", las cuales ocupan un lugar secundario en la historia dominante del ser y posibilitan el ejercicio de reautoría o de reescribir la vida (White, M. 2004; Morgan, A. 2000).

“La estructuración de una narración requiere la utilización de un proceso de selección por medio del cual dejamos de lado, de entre el conjunto de los hechos de nuestra experiencia, aquellos que no encajan en los relatos dominantes que nosotros y los demás desarrollamos acerca de nosotros mismos" (Epston y White, 1993: 29). Entonces los elementos que "se dejan de lado" al momento de contar una historia, abren un campo de acción e indagación terapéutica, en el cual emergen posibilidades o logros aislados, como lo han denominado los autores. Los logros aislados posibilitan integrar a la trama dominante y saturada del problema, otros aspectos, los cuales la persona no los tenía integrados. De esta manera, al engrosar la trama dominante, emergen nuevas historias, nuevos logros, los cuales se encuentran enraizados en nuestra cultura, valores y creencias.

\section{Relato, Conocimiento y Poder}

En el contexto de las prácticas narrativas, la importancia de reconocer el poder y conocimiento en una relación indisoluble, en tanto un elemento es constituyente de la otra. De igual forma, desde estos planteamientos, no podría ser posible la existencia del ejercicio del poder sin una cuota importante de "discursos de verdad"; las personas estaríamos sujetas a la producción y reproducción de discursos de verdad a través del poder, y no podemos ejercitar el poder si no es a través de una producción de verdad (Foucault, citado en Epston y White, 1993).

Las verdades "objetivas" que se generan a través de los actos performativos en el enlazamiento poder/conocimiento suelen generar prácticas sociales específicas, en tanto posibilitan un marco inteligible, comprensivo y explicativo de los fenómenos que se estudian o pueden estudiarse. Al mismo respecto, podemos ejemplificar ello con la homosexualidad y cómo se ha entendido, expli- 
cado y comprendido dicha acepción. Prosiguiendo con las ideas anteriores, en el DSM-II ${ }^{18}$ (1968-1973) se contempla la homosexualidad como una enfermedad o afectación psicopatológica. Por ende, debía ser tratada con los métodos de la época ${ }^{19}$. Como vemos, un tipo de conocimiento: el científico-médico concentrado en la psiquiatría como disciplina, informaba al público, mediante el poder que le otorgaba su estatus, que la homosexualidad era una enfermedad psicológica grave.

Conforme avanzó el tiempo, esta comunidad "científica" decide eliminar la homosexualidad como trastorno grave de la conducta sexual humana. Es por ello que, a partir del DSM-III en adelante, dicha patología de la mente no existió más. Ahora bien ¿qué sucedió con todas las personas que se les trató como enfermas de homosexualidad hasta 1973? Dicha comunidad científica denominada psiquiatría no ha reparado aquel daño perpetrado bajo las nociones de verdad objetiva, de enfermedad y de discursos - COnocimientos-, cargados de poder científico. Al respecto se construyeron nociones de verdad, las cuales objetivaron, cosificaron, catalogaron, excluyeron y sometieron a una serie de individuos, tornando sus cuerpos, en "cuerpos dóciles", en palabras de Foucault (citado en Epston y White, 1993).

En este sentido, las historias que se cuenta a sí mismo una persona, como las historias que los otros cuentan de él o ella, tienen implicancia directa en el sujeto. Las historias moldean las vidas de las personas; ello es posible, en tanto el conocimiento y poder se encuentran indefectiblemente relacionados, por lo tanto, son los efectos de ese poder-conocimiento los que van moldeando lentamente a las personas. Los efectos experimentados no son los que comúnmente podríamos argumentar, los efectos descalificadores, limitantes, negadores o coactivos (Epston y White, 1993). Por el contrario, son los efectos positivos los que afectan a la persona, los efectos de "verdades normalizadoras", las cuales configuran las vidas de las personas y las relaciones de éstas. Estas "verdades", a su vez se constituyen o producen en el funcionamiento del poder (Foucault, 1979, 1980, 1984a,. citado en y Epston y White, 1993).

18 Manual diagnóstico y estadístico de los trastornos mentales. En inglés: Diagnostic and Statistical Manual of Mental Disorders

19 Principalmente destacan los modelos terapéuticos basados en la reconversión, reorientación e incluso electro shock. 


\section{La Imposibilidad de Acceder a la Realidad}

El primer aspecto epistemológico fundamental en terapia narrativa, retrotrae a los aportes de Gregory Bateson, en relación a la imposibilidad de conocer o tener acceso a una realidad "objetiva", ya que todo conocimiento requiere un acto de interpretación (Epston y White, M., 1993).

A este respecto, la posición del sujeto que conoce no se encuentra fuera del sistema observado, por el contrario, se encuentra inmerso en las observaciones y distinciones que realiza, en las cuales participa activamente de la construcción del objeto observado (Keeney, B., 1994).

Por otra parte, siguiendo los planteamientos de Maturana y en concordancia con la imposibilidad de acceder a una realidad, la objetividad entre paréntesis ${ }^{20}$ plantea que la realidad que observa un observador, no se encuentra fuera de él, no existe con independencia de lo que se haga o deje de hacerse, en la (objetividad) "el observador sabe que él o ella no puede usar un objeto que se asume existe como una entidad independiente como un argumento para fundar su explicación" (Maturana, 2011: 24) En este sentido, argumentar que existe una realidad accesible a todas las personas, independiente de quien la observa, no sería más que un argumento para obligar.

\section{Reflexiones finales}

La práctica narrativa ${ }^{21}$ expone que el término "terapia" es un concepto inadecuado para describir el trabajo que se realiza desde esta práctica. En el entendimiento que el concepto de terapia aduce al tratamiento de enfermedades, desórdenes, defectos, la práctica narrativa no entiende los problemas en términos de enfermedades, defectos o déficits y no cree en nada que pueda relacionarse con una "curación" (White, 1990). Existe una visión despatologizadora, no centrada en el diagnóstico. Lo que no quiere decir que se invalide o se desconozca lo anteriormente

\footnotetext{
20 Concepto que utiliza el autor para hacer referencia a la existencia de una realidad observada por un observador, el cual está inmerso en el sistema observado, no se encuentra ni se encontrará fuera del sistema observado.

21 La terapia narrativa inicialmente es conocida como un enfoque, luego se posiciona como terapia y posteriormente es denominada como práctica narrativa (White, 2007).
} 
mencionado, sino que el diagnóstico o la patología, es parte de una historia más amplia y, en ningún caso, la patología o el diagnóstico va a definir a la persona.

Entonces, aparece la estrategia fundamental en Narrativa, la externalización. La externalización del problema es la técnica terapéutica central usada por Michael White para ayudar a las personas a comenzar a definir sus problemas como separados de sus identidades. Para ello desarrolla un estilo particular de entrevista, preguntando acerca de cómo el problema ha estado afectando la vida de la persona y sus relaciones. Lo cual posibilita la identificación de los aspectos problemáticos y cuándo se ha tenido el control sobre ellos. De igual forma, otorga el control a la persona sobre el problema y no a la inversa, que el problema es quien gobierna sus vidas. Lo que se busca como fin último es la recuperación de la agencia personal.

Respecto de la posición de las personas como agentes en sus propias vidas, Michael White y David Epston realizan una comparación acerca de la representación del modo lógico científico y del modo narrativo acerca de esta visión del ser humano. Para estos autores, el modo lógico científico representa a la persona como pasivo/a frente a fuerzas internas y externas que moldean sus vidas (Impulsos, impactos, transferencias, etc.).

En cambio el modo narrativo sitúa a la persona como protagonista de su propio mundo o historia. Es un mundo interpretativo en donde recontar una historia es contar una historia nueva, generando nuevos significados a medida que la persona con sus semejantes van reescribiendo y moldeando sus vidas y relaciones (Epston, D. y White, M., 1993).

"Esta revisión ética (...) se dirige enseguida a los riesgos de privilegiar el micromundo de la terapia por encima de los macrocontextos de las vidas de las personas" (White, 2002: 237). White, a este respecto, alude a un punto específico: el desaliento de los terapeutas, en relación con las ideas de acción efectiva en la relación terapeuta-consultante. Lo cual se relaciona indefectiblemente con un contexto sociopolítico más amplio, el cual establece-mediante diversos mecanismos-, las formas y tiempos de atención, por lo general breves en su duración, con exigencias de logros exitosos y márgenes de error exiguos; sobre ello, la idea de 
acción efectiva, se vincula directamente con la ética del control (White, M., 2002).

Si bien en un inicio White anuncia lo riesgoso de concentrarse en los aspectos macrocontextuales en terapia, de igual forma lo es centrarse en aspectos microcontextuales, en tanto en ese espacio es el terapeuta quien tiene el mayor poder conceptual para "dominar" la sesión a su antojo; de esta forma, se invisibiliza al consultante (White, M., 2002). Como contraparte a ello, el mismo autor explicita una posible disolución a la coyuntura antes mencionada, en la cual se propone ejercer una práctica descentrada pero influyente en la relación terapeuta-consultante, para la cual enuncia que la importancia de las conversaciones de re-integración pongan el acento en los aprendizajes del consultante; que la valoración de la narración y la re-narración posibilite la aparición de nuevas narraciones enriquecidas y ellas se integren a la historia dominante; que se incorpore la participación de testigos externos, los cuales contribuyen a validar la experiencia del otro. Desde esta perspectiva (en contraposición a la perspectiva del terapeuta); validar la experiencia del otro y cómo ello ha contribuido a moldear su experiencia y su operar en el mundo, es vital.

Los planteamientos anteriormente expuestos son sumamente importantes en terapia narrativa, en tanto, existe un reconocimiento de la experiencia de la persona, experiencia que lo constituye como tal y que permite un reconocimiento del otro, una validación de la experiencia y la posibilidad de deconstruir dichas experiencias a partir de las narrativas subjetivas del consultante.

\section{Epítome}

Es una tarea compleja poder finalizar el presente artículo, expresando opiniones propias respecto de la redacción del mismo. Difícil tarea, pues, intentar resumir y abarcar a la vez, la gran mayoría de los modelos imperantes en trabajo social, supone retrotraer al presente artículo casi 100 años de historia ${ }^{22}$, sobre ello, la elección del modelo narrativo o, mejor dicho, las prácticas narrativas,

\footnotetext{
Si consensuamos que, uno de los eventos que marcaría el inicio de la profesión es la publicación del libro "Diagnóstico Social" en el año 1917, cuya autora es Mary Richmond, aunque no existe una referencia tácita al inicio del Trabajo Social, en tanto se venían desarrollando iniciativas de esa índole desde mucho antes y la disciplina fue formalizada en el campo universitario después de 1917.
} 
supone la revisión de un modelo reciente, cuya literatura nos fue accesible hace un poco más de 20 años (1991-1993). La importancia de co-construir prácticas de trabajo desde la narrativa, supone relevar la agencia personal de las personas con las cuales trabajamos, las personas son las expertas en sus propias vidas, no el trabajador o trabajadora social -como ocurre en otros modelos, en los que el profesional es el experto-. Desde la narrativa, interesan las historias, todas las historias, las dominantes o cargadas de problemas y sobre todo, las subyugadas o relegadas a un lugar secundario o terciario; de ahí emergerán posibilidades de cambio. De igual modo, es una práctica que incluye con fuerza el contexto social, político y cultural. De esta forma, la vida de la persona no se encuentra inconexa con su medio circundante, el cual influye directa y poderosamente en la vida de las personas.

La posibilidad de trabajo colectivo, con grupos o comunidades que han experimentado situaciones traumáticas o bien, aquejados por las profundas desigualdades de nuestra sociedad. Son objeto también de las prácticas narrativas, mediante ellas podemos tejer y relatar nuevas historias de sobrevivencia a la opresión, exclusión o tortura, por ejemplo. Rescatar los actos de resistencia frente a las injusticias y no quedarnos con ello solamente, sino, compartir las experiencias comunitarias con otras comunidades que experimenten situaciones similares o totalmente distintas.

Hay que tener presente que los modelos en trabajo social han sido influenciados poderosamente por los cambios en las distintas ciencias y, sobre todo, en las ciencias sociales, siendo la psicología la que ha iluminado algunos modelos, como por ejemplo: intervención en crisis, centrado en tareas o en soluciones, entre otros. De igual forma, estos modelos han transitado desde el positivismo más clásico, cuya traducción a las ciencias sociales nos da una visión del ser humano estática, determinada por sus condicionantes internos y con pocas posibilidades de cambio. Luego, con la emergencia de la teoría general de sistemas, lo humano pasó a la relación, dejando de lado -por un buen tiempo-, lo individual. Después, el constructivismo nos brindó la posibilidad de poner al ser humano en el centro de las construcciones sociales e individuales en las cuales participa activamente.

Finalmente, posiciona a la persona como agente de su propia vida, no como un agente que "debe" acomodarse a los intereses 
del grupo o a los intereses del mercado. Al estar sentado en el posmodernismo, la noción de verdad y objetividad carecen de total sentido, ya no hay verdades o lugares finales a los cuales llegar, cada persona encontrará por sí sola o en compañía de otros, un lugar y su propia verdad.

\section{Bibliografía}

Aylwin, N. y Solar, O. (2002). Trabajo Social Familiar, Santiago de Chile: Ediciones Universidad Católica de Chile.

Bateson, G. (1976). Pasos Hacia una Ecología de la Mente. Buenos Aires: Carlos Lohlé.

Campanini, A y Luppi, F. (1991). Servicio Social y modelo sistémico, Una nueva Perspectiva para la práctica. Barcelona, España: Paidós.

Demichelli, G. (1995). Comunicación en terapia familiar sistémica. Valparaíso, Chile: Universidad de Valparaíso.

De Robertis, C, y Pascal, H. (2006). La Intervención Colectiva en Trabajo Social, la acción con grupos y comunidades. Buenos Aires, Argentina: Hvmanitas.

De Robertis, C. (1994). Metodología de la Intervención en Trabajo Social. Buenos Aires, Argentina: Hvmanitas.

Epston, D. y White, M. (1993). Medios narrativos para fines terapéuticos, Barcelona, España: Paidós.

Keeney, B. (1994). Estética del Cambio. Buenos Aires, Argentina: Paidós.

Matus, T. (1999). Propuestas Contemporáneas en Trabajo Social, Hacia una Intervención Polifónica. Buenos Aires, Argentina: Espacio Editorial.

Morán, J. (2003). Epistemología, Ciencia y Paradigma en Trabajo Social. Sevilla, España: Aconcagua Libros.

Maturana, H. (2011). La Objetividad, Un Argumento para Obligar. Santiago de Chile: Dolmen Ensayo.

Morgan, A. (2000). What Is Narrative Therapy?, Dulwich Centre Publications, Adelaide, South Australia. Recuperado de: http://www.dulwichcentre.com.au/what-isnarrative-therapy.html

Pérez, J. (1984). Terapia Familiar en el Trabajo Social. Teoría y práctica. México: Editorial Pax. 
Quintero, A. (2004). Trabajo Social y Procesos Familiares (Segunda edición). Buenos Aires, Argentina: Editorial Lumen/Humanitas.

White, M. (2004). Guías para una Terapia Familiar Sistémica ( $2^{\circ}$ Reimpresión). Barcelona, España: Gedisa.

White, M. (2002). El Enfoque Narrativo en la Experiencia de los Terapeutas. Barcelona, España: Gedisa. 
